\title{
Water in oil microemulsions : transient electric birefringence response
}

\author{
P. Guering and A. M. Cazabat \\ Laboratoire de Spectroscopie Hertzienne de l'E.N.S., 24, rue Lhomond, 75231 Paris Cedex 05, France
}

(Reçu le 6 avril 1983, accepté le 19 mai 1983)

\begin{abstract}
Résumé. - Nous avons étudié la structure de microémulsions eau dans huile au moyen de la biréfringence électrique transitoire. A faible concentration, on observe l'association des gouttelettes, à concentration moyenne des fluctuations de densité dans le système. Une composante négative, rapide, est attribuée aux fluctuations des interfaces. Une étude plus complète suivra ces résultats préliminaires.
\end{abstract}

\begin{abstract}
The structure of water in oil microemulsions has been investigated using transient electric birefringence. At low water concentrations, transient coalescence of droplets is observed. At higher water concentrations, the Kerr signal probes density fluctuations. A fast negative response is attributed to the relaxation of interfacial layers. Further work is under way to make these preliminary results more quantitative.
\end{abstract}

\section{Introduction.}

Microemulsions [1] are transparent dispersions of oil and water, stabilized by the presence of surfactant.

At low water content, X-ray and neutron scattering experiments [2] indicate that water in oil (W/O) microemulsions are composed of dilute monodisperse spherical water droplets, dispersed in a continuous oil phase.

Each droplet is sheathed by a film of amphiphilic molecules. A short chain alcohol plays the rôle of stabilizing agent and is partitioned between the droplet interfaces and the continuous phase [3].

At higher volume fractions, $\phi$, of droplets scattering experiments cannot give unambiguous information about structure, because of the occurrence of interactions. At the present time, at large $\phi$ values the structure of the medium is still conjectural.

We report here a transient electric birefringence [4] study of the structure of microemulsions. Electric and magnetic birefringence have already been used to study lamellar phases and colloidal systems near phase separation [5-9].

The techniques involve the application of steady electric or magnetic fields which orient the macroscopic structure, in some cases deforming it also. Orientated anisotropic structures can be elongated droplets, aggregates, flexible interfaces [10], or lamellae. 
For transient experiments, the amplitude and time rate of change of the associated optical birefringence is followed when the fields are turned on or off. From this information, some aspects of the nature of the orientated anisotropic structures can be inferred.

\section{Materials and method.}

- Seven series of microemulsions located on demixion lines of their phase diagrams were studied. Along a demixion line, it is possible to vary the volume fraction $\phi$ of droplets while keeping their size constant.

For each series, the droplet radius $R$ and the second virial coefficient $\beta$ of the osmotic compressibility were measured by quasielastic light scattering. The results are reported in table I. A detailed study of most systems listed in table I has already appeared [11].

Table I. - W/O microemulsions systems.

\begin{tabular}{|l|c|c|c|c|c|}
\hline Code & Surfactant & Oil & Alcohol & $\begin{array}{c}\text { Droplet } \\
\text { radius } \\
\AA\end{array}$ & $\beta$ \\
\hline ATP & SDS & T & P & 45 & +7 \\
BCP & SDS & C & P & 81 & -1 \\
$\alpha$ TB & SDS & T & B & 36 & -12 \\
ATB & SDS & T & B & 41 & -20 \\
$\beta T B\left(^{*}\right)$ & SDS & T & B & 60 & -30 \\
ACB (*) & SDS & C & B & 48 & $\lesssim-40$ \\
$\gamma$ TB (*) & SDS & T & B & 90 & $\lesssim-40$ \\
\hline
\end{tabular}

SDS sodium dodecyl sulfate, $\mathbf{T}$ toluene, $\mathrm{C}$ cyclohexane, $\mathrm{P}$ pentanol, B butanol.

$\left(^{*}\right)$ For $\beta \mathrm{TB}, \mathrm{ACB}$ and $\gamma \mathrm{TB}$ microemulsions, the droplet size can be kept constant only for $\phi \lesssim 0.03$.

Note that for hard sphere-like droplets, the virial coefficient $\beta$ would be +8 . The negative $\beta$ values observed in all cases but one indicate the occurrence of strong attractive interactions [12] between droplets.

- The experimental set-up was classical [4, 13]. Linear detection [14] was used because it affords high sensitivity. Thus relatively low electric fields could be applied, minimizing the thermal disturbance of the samples.

Pulse amplitudes varied from $30 \mathrm{~V} / \mathrm{cm}$ to $4 \mathrm{kV} / \mathrm{cm}$ (for very low $\phi$ ), pulse durations from $0.1 \mu \mathrm{s}$ to $100 \mathrm{~ms}$. Rise and fall times were less than $30 \mathrm{~ns}$. Signals were averaged up to 256 times using a digital storage oscilloscope.

The steady state induced birefringence $\Delta n$ is proportional to the square of the electric field $E$

$$
\Delta n=B \lambda E^{2},
$$

here $\lambda$ is the wavelength of the light beam $(\lambda=514 \mathrm{~nm})$ and $B$ is the Kerr constant of the sample.

The value of $\Delta n$ was typically about $10^{-9}$. 


\section{Experimental results.}

3. 1 Low CONCENTRATIONS. - Figure 1 shows the results of measurements performed at very low droplet concentrations $\left({ }^{1}\right)$. The Kerr constant $B$ is plotted against the droplet volume fraction $\phi$. The system codes are identified in table I. The Kerr constant $B_{\text {cont }}$ of the continuous phase has been subtracted for each series to avoid curves crossing. (For example : $B_{\text {cont }}=$ $+45 \times 10^{-16} \mathrm{mV}^{-2}$ for the ATP series, $+33 \times 10^{-16} \mathrm{mV}^{-2}$ for the $\beta$ TB series. Note that $B=+76 \times 10^{-16} \mathrm{mV}^{-2}$ for toluene [15]). All measured Kerr constants are positive. The curves in figure 1 are curved everywhere. This implies that the induced birefringence is not due to orientation of individual elongated droplets or of elements of their interfacial films : these processes would give a linear dependence of $B$ upon $\phi$, at least for small $\phi$ (for instance, less than $5 \%$ ). (Note also that the intrinsic viscosities of all the microemulsions are close to 2.5 [16], the value expected for spherical particles).

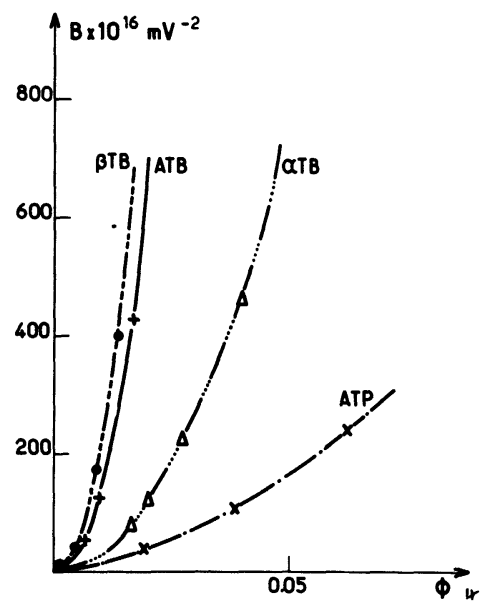

Fig. 1. - Kerr constant $B$ versus volume fraction $\phi$ for several microemulsion series.

It seems likely that the induced birefringence is due to the reorientation of short-lived aggregates which form in the course of sticky collisions between droplets [17].

It has been observed [18] that the probability of sticky collisions increases rapidly with the strength of the attractive potential between droplets. Indeed, the measured Kerr constants are very well correlated with the virial coefficient $\beta$ (cf. table I) of the osmotic compressibility.

Examination of the decay of the birefringence reveals non exponential behaviour, with several characteristic times in the range 1-10 $\mu \mathrm{s}$. This is again in good agreement with the picture of reorienting transient aggregates of various sizes. By comparison, the characteristic time $\tau$ for one spherical droplet of radius $R=50 \AA$ is [4]

$$
\tau=\frac{8 \pi \eta R^{3}}{6 k T}=70 \mathrm{~ns},
$$

here $\eta$ is the microemulsion viscosity, $\eta=2 \mathrm{cp}$.

A quantitative analysis of the decay curves is underway.

3.2 INTERMEDIATE CONCENTRATIONS. - Figure 2 displays the logarithm of the Kerr constant $B$ versus the volume fraction $\phi$ of the dispersed phase at intermediate concentrations. For all the systems studied, the curves go through a maximum.

( ${ }^{1}$ In this range, the microemulsion is known to be a dispersion of droplets [2]. 
This maximum coincides with the maximum of the microemulsion turbidity measured by light scattering [11].

The mean decay time roughly follows the $B$ variation.

The ATB series passes close to a critical demixion point for $\phi=0.08$ [19]. Percolation of the electric conductivity is also observed at this volume fraction. This is displayed in figure 2 which includes a plot of the logarithmic electric conductivity $K$ versus $\phi$ for the ATP and ATB systems. The measured $B$ values are not immediately, interpretable : indeed, dielectric relaxation probably occurs at $\phi \sim 0.08$, as already observed near demixion points in microemulsions [20, 21]. A local field correction is thus necessary [22]. Detailed analysis of figure 2 is thus not attempted at this time.

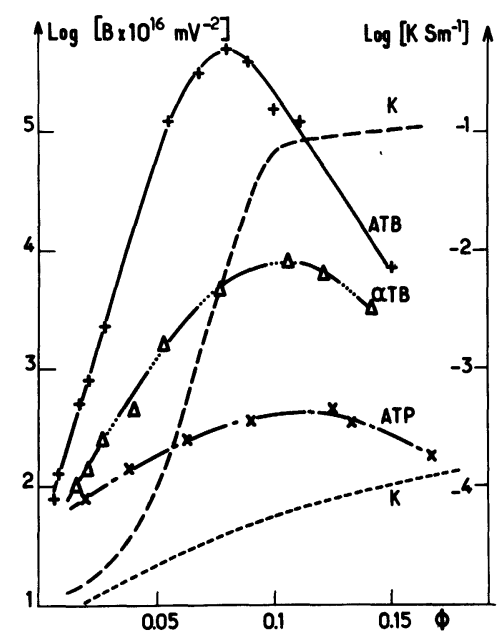

Fig. 2. - Plots of logarithmic Kerr constant $B$ (left scale) and electric conductivity $K$ (right scale) versus $\phi$, for ATB (- - $)$ and ATP (-----) systems.

However, quantitative results concerning the time decay of the birefringence can be obtained for the ATB system at $\phi \sim 0.08$. It is well fitted by a single exponential, whose characteristic time $\tau$ is in fairly good agreement with the lifetime $\tau_{\xi}$ of a critical density fluctuation of « size » $\xi$. $\xi$, the correlation length of the density fluctuations, has already been measured for the ATB microemulsion by light scattering [19]. $\tau_{\xi}$ is related to $\xi$ through the formula :

$$
\tau_{\xi}=\frac{6 \pi \eta \xi^{3}}{k T}
$$

Comparison of the calculated $\left(\tau_{\xi}\right)$ and measured $(\tau)$ values is given in table II.

Table II.

\begin{tabular}{|c|c|c|c|}
\hline$\phi$ & $\xi\left(^{*}\right)(19) \AA$ & $\tau_{\xi}(\mu \mathrm{s})$ & $\tau(\mu \mathrm{s})$ \\
\hline 0.07 & 240 & 120 & 150 \\
0.08 & 280 & 190 & 230 \\
0.09 & 300 & 230 & 200 \\
\hline
\end{tabular}

Calculated $\left(\tau_{\xi}\right)$ and measured $(\tau)$ values of the birefringence decay time.

(*) $T=19{ }^{\circ} \mathrm{C}$. 
Although the variations of the Kerr constants are very large in the intermediate concentration range, they cannot be related directly to the microemulsions structures. For example, birefringent lobes exist in many phase diagrams close to the isotropic microemulsion domain, especially in the vicinity of critical points [19]. Could the Kerr signal probe pretransitional processes ? More detailed analysis of the time decay of the birefringence may answer this question, but such analysis is not attempted at the present time.

3.3 LARGE CONCENTRATIONS. - At larger water concentrations, a somewhat surprising observation was made : a fast, negative contribution to the birefringence signal arises and grows rapidly with $\phi$. In figure 3 , the Kerr constants of the positive and negative contributions $\left({ }^{2}\right)$ are plotted against the volume fraction $\phi$ for the $\alpha$ TB system. In the ATB system, which cannot be studied at $\phi$ greater than $15 \%$ because of its large electrical conductivity, this process was not observed.

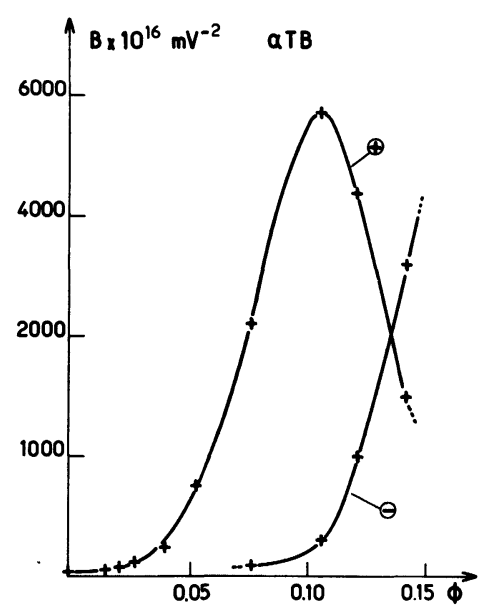

Fig. 3. - Kerr constant $B$ versus volume fraction $\phi$ for $\alpha$ TB microemulsions : $\oplus$ positive contribution, $\ominus$ negative contribution.

Figure 4 shows that the negative contribution is very important, even at low concentrations, in microemulsions where the oil is cyclohexane.

The short $(1 \mu \mathrm{s})$ decay times suggest that the process reflects local reorganization of the interfacial film [10]. Such time scales correspond to orientational fluctuations of flexible interfaces characterizing microemulsion systems [23]. Similar behaviour (but with larger characteristic times) has already been observed in polymer systems [24]. The slow positive effect [25] was attributed to polymer chain reptation [26], the fast negative effect to local reorganization of the chain.

This qualitative picture agrees well with the large negative values measured in the vicinity of the inversion point (cf. Fig. 4). Moreover, exchanges through the interfacial film are known to be very important in microemulsions containing cyclohexane. NMR [27] and ultrasonic absorption [28] show that the exchanges are less if the oil is toluene or benzene (compounds with benzene ring).

$\left({ }^{2}\right)$ As the decay times of the two contributions are very different $(1: 10)$, it is possible to measure each of them separately. 


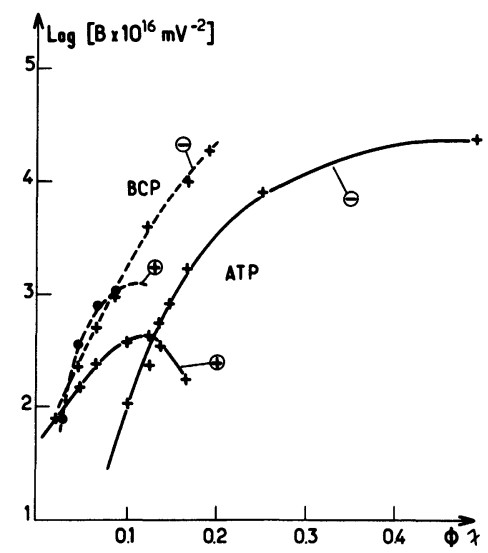

Fig. 4. - Plot of logarithmic Kerr constant $B$ versus volume fraction $\phi$ for ATP and BCP microemulsions : $\oplus$ positive contribution, $\Theta$ negative contribution.

\section{Summary and conclusion.}

Transient electric birefringence is a promising method for obtaining structural information about microemulsions systems. At present, only qualitative conclusions can be drawn. At very low water concentrations, the effects of transient association of droplets are observed. At intermediate volume fractions of water, the birefringence signal is related to density fluctuations. At high concentrations, the birefringence reflects local reorganization of the interfacial layer.

Further work is needed to make these conclusions quantitative. A detailed analysis of the time decay of the birefringence is under way.

\section{Acknowledgments.}

Fruitful discussions with M. Paillette (Groupe de Physique des Solides de l'Ecole Normale Supérieure), D. Chatenay, D. Langevin and J. Meunier are gratefully acknowledged.

We wish to thank S. Granick for his critical reading of the manuscript.

\section{References}

[1] Hoar, T. P., Schulman, J. H., Nature 102 (1943) 152.

[2] Dvolaitzky, M., Guyot, M., Lagües, M., Lepesant, J. P., Ober, R., Sauterey, C., Taupin, C., J. Chem. Phys. 69 (1978) 3279.

[3] Schulman, J. H., Stoeckenius, W., Prince, L., J. Phys. Chem. 63 (1959) 1677.

[4] Benort, H., Ann. Phys. 6 (1951) 561.

[5] Eicke, H. F., Markovic, Z., J. Colloid Interface Sci. 79 (1981) 151.

[6] Eicke, H. F., MARKovic, Z., J. Colloid Interface Sci. 85 (1982) 198.

[7] Hoffman, R., International Symposium on Surfactants in solution, Lund (Sweden) 1982, to be published.

[8] Nicoli, D. F., Elias, J. G., Eden, D., J. Phys. Chem. 85 (1981) 2866.

[9] Meyer, C. T., Poggi, Y., Maret, G., J. Physique 43 (1982) 827.

[10] Jouffroy, J., Levinson, P., DE Gennes, P. G., J. Physique 43 (1982) 1241.

[11] Cazabat, A. M., Langevin, D., J. Chem. Phys. 74 (1981) $3148 ; \beta$ TB and $\gamma$ TB microemulsions have been studied by $D$. Chatenay (unpublished results).

[12] Lemaire, B., Bothorel, P., Roux, D., to be published. 
[13] Molecular Electrooptics, C. T. O’Konski, ed. (Marcel Dekker, Inc. New York and Basel) 1976.

[14] BAdoz, J., J. Physique Radium 17 (1956) 143A.

[15] Beevers, M. S., Khanarian, G., Aust. J. Chem. 32 (1979) 263.

[16] Chatenay, D., unpublished results.

[17] Eicke, H. F., ShePherd, J. C. W., Steineman, A., J. Colloid Interface Sci. 56 (1976) 168.

Robinson, B. H., Steytler, D. C., TACK, R. D., J. Chem. Soc. Faraday Trans. 175 (1979) 781 ; Ober, R., Taupin, C., J. Phys. Chem. 84 (1980) 2418.

[18] International Symposium on Surfactants in solution, Lund (Sweden) 1982. Discussions.

[19] Cazabat, A. M., Langevin, D., Sorba, O., J. Physique Lett. 43 (1982) L-505.

[20] Eicke, H. F., ShePherd, J. C. W., Helv. Chim. Acta 57 (1974) 1951.

[21] Senatra, D., Giubilaro, G., J. Colloid Interface Sci. 67 (1978) 448.

[22] Palllette, M., private communication.

[23] Di Meglio, J. M., communication at the Gripp meeting of Greco "Microemulsions ";

Di Meglio, J. M., Dvolaitzky, M., Ober, R., TAupin, C., J. Physique Lett. 44 (1983) L-229.

[24] Beevers, M. S., Elliott, D. A., Williams, G., Polymer 21 (1980) 13.

[25] DoI, M., J. Polymer Sci. 20 (1982) 1963.

[26] De Gennes, P. G., J. Chem. Phys. 55 (1971) 572.

[27] Sü̈blom, E., Henriksson, U., J. Phys. Chem. 86 (1982) 4451.

[28] Zana, R., Lang, J., Sorba, O., Cazabat, A. M., Langevin, D., J. Physique Lett. 43 (1982) L-829. 